\title{
OPEN
}
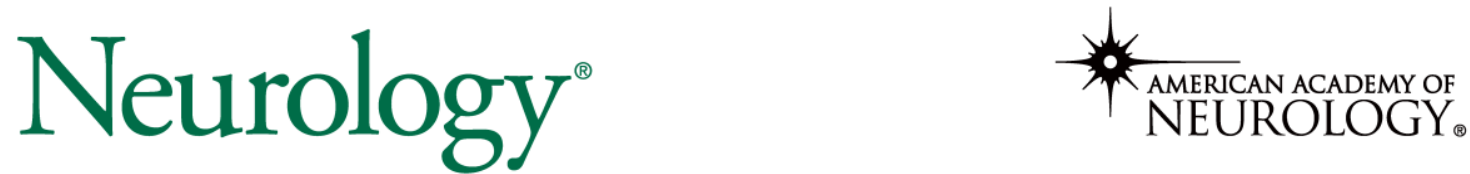

The most widely read and highly cited peer-reviewed neurology journal

The Official Journal of the American Academy of Neurology

Neurology Publish Ahead of Print DOI: 10.1212/WNL.0000000000200109

\section{Endoluminal Biopsy for Molecular Profiling of Human Brain Vascular Malformations}

\section{Author(s):}

Ethan Andrew Winkler, MD, $\mathrm{PhD}^{1}$; David Wu, BS ${ }^{1}$; Eugene Gil, BS ${ }^{1}$; David McCoy, $\mathrm{MS}^{2}$; Kazim

Narsinh, $\mathrm{MD}^{2}$; Zhengda Sun, $\mathrm{PhD}^{2}$; Kerstin Mueller, $\mathrm{PhD}^{3}$; Jayden Ross, $\mathrm{BA}^{1,4}$; Helen Kim, $\mathrm{PhD}^{5}$; Shantel Weinsheimer, $\mathrm{PhD}^{5}$; Mitchel Berger, $\mathrm{MD}^{1}$; Tomasz Nowakowski, $\mathrm{PhD}^{4,6,7}$; Daniel Lim, MD, PhD ${ }^{1,7}$; Adib Abla, MD ${ }^{1}$; Daniel Cooke, $\mathrm{MD}^{2}$

This is an open access article distributed under the terms of the Creative Commons Attribution-NonCommercial-NoDerivatives License 4.0 (CC BY-NC-ND), which permits downloading and sharing the work provided it is properly cited. The work cannot be changed in any way or used commercially without permission from the journal.

Neurology ${ }^{\circledR}$ Published Ahead of Print articles have been peer reviewed and accepted for publication. This manuscript will be published in its final form after copyediting, page composition, and review of proofs. Errors that could affect the content may be corrected during these processes. 
Equal Author Contributions:

A.A.A and D.L.C are co-corresponding authors

\author{
Corresponding Author: \\ Daniel Cooke \\ daniel.cooke@ucsf.edu
}

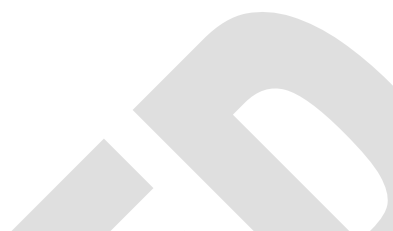

Affiliation Information for All Authors: 1. Department of Neurological Surgery, University of California San Francisco, San Francisco, CA, USA; 2. Department of Radiology and Biomedical Imaging, University of California San Francisco, San Francisco, CA, USA; 3. Siemens Medical Solutions Inc., Malvern, PA; 4. Department of Anatomy, University of California San Francisco, San Francisco, CA, USA, Chan Zuckerberg Biohub, San Francisco, CA, USA; 5. Center for Cerebrovascular Research, University of California San Francisco, San Francisco, CA, USA; 6. Departments of Psychiatry and Behavioral Sciences, University of California San Francisco, San Francisco, CA; 7. The Eli and Edythe Broad Center for Regeneration Medicine and Stem Cell Research, University of California San Francisco, San Francisco, CA

\title{
Contributions:
}

Ethan Andrew Winkler: Drafting/revision of the manuscript for content, including medical writing for content; Major role in the acquisition of data; Study concept or design; Analysis or interpretation of data David Wu: Major role in the acquisition of data

Eugene Gil: Major role in the acquisition of data

David McCoy: Analysis or interpretation of data

Kazim Narsinh: Drafting/revision of the manuscript for content, including medical writing for content; Major role in the acquisition of data

Zhengda Sun: Major role in the acquisition of data; Analysis or interpretation of data

Kerstin Mueller: Analysis or interpretation of data

Jayden Ross: Major role in the acquisition of data

Helen Kim: Drafting/revision of the manuscript for content, including medical writing for content; Study concept or design

Shantel Weinsheimer: Drafting/revision of the manuscript for content, including medical writing for content; Study concept or design

Mitchel Berger: Study concept or design

Tomasz Nowakowski: Drafting/revision of the manuscript for content, including medical writing for content; Study concept or design

Daniel Lim: Drafting/revision of the manuscript for content, including medical writing for content; Study concept or design

Adib Abla: Drafting/revision of the manuscript for content, including medical writing for content; Study concept or design

Daniel Cooke: Drafting/revision of the manuscript for content, including medical writing for content; Study concept or design

Number of characters in title: 71

Abstract Word count: 299

Word count of main text: 3925

References: 42

Figures: 4 
Tables: 1

Supplemental: Supplemental Figure 1 Supplemental Table 1-2Supplemental Video 1-2 Strobe checklist

Search Terms: [ 3 ] Arteriovenous malformation, [ 7 ] Intracerebral hemorrhage, [ 11 ] Stroke in young adults, [ 304 ] Gene expression studies

Acknowledgements: The authors thank Kenneth Xavier Probst for illustrations in Figure 1.

Study Funding: EAW is supported by a Brain Vascular Malformation Consortium (BVMC) Pilot Feasibility Project Grant and the Cynthia Lynn Sherwin and Buzz for BAF Chair of Research grant from the Brain Aneurysm Foundation. The BVMC (U54NS065705) is a part of the NCATS Rare Diseases Clinical Research Network (RDCRN) and is supported by the RDCRN Data Management and Coordinating Center (DMCC) (U2CTR002818). DLC is supported through the UCSF Department of Radiology and Biomedical Imaging, UCSF REAC grant and Siemens Healthineers AG. KHN is supported by the BVMC Early Career Enhancement Award (U54NS065705).

Disclosures: The authors report no disclosures relevant to the manuscript.

\section{Abstract}

Background and Objectives: Ras-mitogen activated protein kinase (Ras-MAPK) signaling abnormalities occur in most brain arteriovenous malformations (bAVMs). No means exist to molecularly profile bAVMs without open surgery, limiting precision medicine approaches to treatment. Here, we report the first in-human use of endoluminal biopsy of the vessel lumen of bAVMs to characterize gene expression and blood-flow mediated transcriptional changes in living patients.

Methods: Endoluminal biopsy and computational fluid dynamic modeling (CFD) was performed in adults with unruptured AVMs with cerebral angiography. Each patient underwent surgical resection and cell sampling from a contiguous arterial segment. Fluorescence-assisted cell 
sorting enriched endothelial cells which were sequenced on an Illumina HiSeq 4000 sequencer. Gene expression was quantified with RNA sequencing (RNAseq). Differential gene expression, ontology and correlative analyses performed. Results were validated with quantitative reverse transcription polymerase chain reaction (RT-qPCR).

Results: Endoluminal biopsy was successful in 4 patients without complication. Endoluminal biopsy yielded $269.0 \pm 79.9$ cells per biopsy (control: $309.2 \pm 86.6$ cells; bAVM: $228.8 \pm 133.4$ ). RNAseq identified 106 differentially expressed genes (DEGs) in bAVMs (FDR $\leq 0.05)$. DEGs were enriched for bAVM pathogenic cascades, including Ras-MAPK signaling ( $p<0.05)$, and confirmed with RT-qPCR and a panel predictive of MAPK/extracellular signal-regulated kinase (MEK) inhibitor response. In comparison to patient-matched surgically excised tissues, endoluminal biopsy detected $83.3 \%$ of genes and genome-wide expression strongly correlated (Pearson $\mathrm{r}=0.77$ ). Wall shear stress measured by CFD correlated with inflammatory pathway upregulation. Comparison of pre-and post-embolization samples confirmed flow-mediated gene expression changes.

Discussion: EB allows molecular profiling of bAVMs in living patients. Gene expression profiles are similar to tissues acquired with open surgery and identify potentially targetable Ras-MAPK signaling abnormalities in bAVMs. Integration with CFD allows determination of flow-mediated transcriptomic alterations. Endoluminal biopsy may help facilitate trials of precision medicine approaches to bAVMs in humans. 
Abbreviations: bAVM, brain arteriovenous malformations; MAPK, mitogen activated kinase; CFD, computational fluid dynamic modeling; DEGs, differentially expressed genes; TPM, transcripts per million; MEK, MAPK/extracellular signal-regulated kinase. 


\section{Introduction}

One type of brain vascular malformation - known as arteriovenous malformations (bAVMs) - are tangles of blood vessels which form direct connections between arteries and veins. ${ }^{1}$ A subset of bAVMs result in intracerebral hemorrhage and are a leading cause of brain hemorrhage in young people. ${ }^{2}$ However, the majority of bAVMs do not hemorrhage.

Randomized controlled trials have suggested that interventional therapy - such as endovascular embolization, stereotactic radiosurgery or surgical resection - are associated with higher rates of death and stroke than observation, and treatment remains controversial. ${ }^{3}$

Precision medicine approaches have revolutionized clinical care - most notably in oncology. Most AVMs were thought to arise without clear genetic cause $(>95 \%)^{4}$, but renewed sequencing efforts have recently identified somatic activating mutations within the Ras-mitogen activated protein kinase (Ras-MAPK) signaling pathway - such as the proto-oncogenes KRAS and $B R A F$ - in a majority of 'sporadic' bAVMs. ${ }^{5,6}$ Targeted inhibition of Ras-MAPK pathways reverses bAVM pathology in experimental models and are being trialed in extracranial vascular malformations. ${ }^{6,7}$ In spite of the ability to identify therapeutic targets, stereotactic brain biopsy techniques are not safe for many cerebrovascular pathologies due to risk of brain hemorrhage. Therefore, molecular profiling is only performed on vascular malformations removed with open surgery. This has several consequences: (1) the information obtained has little influence on clinical decision-making, (2) precision medicine treatment guided by the bAVM's molecular profile is not possible, and (3) no molecular information is available from vascular malformations that are not surgically resected. As a result, there is presently no means to extract molecular information from human cerebrovascular pathologies in living patients. 
To address this barrier to translation of cerebrovascular precision medicine in humans, we sought to use cerebral angiography - the diagnostic gold standard - to sample cells of the vessel lumen from within the cerebrovasculature. Here, we report our case series demonstrating feasibility and accuracy of cerebral angiography-guided endoluminal biopsy to molecularly profile bAVMs with next-generation RNA sequencing (RNAseq), identify therapeutically targetable pathways, and deduce flow-mediated transcriptomic alterations from unruptured bAVMs in living patients.

\section{Methods}

Standard Protocol Approvals, Registrations, and Patient Consents

Human bAVM samples and clinical data were obtained from the University of California San Francisco with protocols approved for human experimentation from the institutional review board and ethics committee (IRB \# 15-16403). In addition to the standard surgical consent, written informed consent was obtained permitting collection of an additional coil during treatment for the specific purpose of research. As part of the institutional review board formal stopping rules were in place, such as a single procedural hemorrhagic or ischemic stroke.

Patients were screened from outpatient clinics and in-hospital census lists. For this initial experience, only adults (>18 years-of-age) with unruptured AVMs undergoing flow-reductive embolization for subsequent microsurgical resection of the AVM were included. Exclusion criteria included AVMs which had previously or currently ruptured, the presence of high-risk features, e.g., flow-related aneurysms, or cerebrovascular anatomy not conducive to catheterization, e.g., vascular tortuosity or unfavorable aortic arch anatomy, or AVMs that were not to be treated with surgical resection. Clinical data is summarized in Table 1. 


\section{Endovascular biopsy}

Digital subtraction angiography (DSA) was performed in standard fashion on either an Artis Q or Artis Zee biplane angiographic system (Siemens Healthineers, Forcheim, Germany). Endovascular biopsy was performed as we have previously described. ${ }^{8,9}$ All patients were administered general anesthesia, and transfemoral access was obtained under ultrasound guidance. After control femoral angiogram, a 0.035-inch Benson or J-curved guide wire was placed through the sheath to contact with the endothelial lining of the iliac artery and kept in this location for $\sim 1$ minute. The wire was removed and the distal $3 \mathrm{~cm}$ were cut and placed into a 3 mL LoBind Eppendorf microcentrifuge tube containing chilled Cell Dissociation Buffer (Thermo Fischer Scientific, Waltham, MA). A post-sampling femoral angiogram was then performed to exclude any procedure-associated arterial dissection. This sample served as a patient-matched control from an uninvolved, systemic artery.

All patients then received an intravenous bolus of heparin to ensure an activated clotting time (ACT) which was $2 \mathrm{x}$ baseline or $>250$ seconds - as this is standard practice to reduce thromboembolic complications with cerebral angiography. A diagnostic cerebral angiogram was then performed with telescoping guide and microcatheters tailored to each patient's cerebrovascular anatomy. Three-dimensional rotation angiography was performed with a Mark7 power injector (MedRad, Beek, Netherlands) to inject the primary arterial supply of the AVM and completely characterize the 3D anatomy of the AVM. A short arterial segment of one of the feeding arterial pedicles was identified a priori as the intended site of the endovascular biopsy. Site selection depended on several selection criteria: (1) the artery was intended to be subsequently embolized for the purposes of reducing flow for surgery the following day; (2) the 
artery could be safely accessed with a microcatheter; (3) the arterial segment was juxtaposed to the AVM nidus (a distance $<\sim 1 \mathrm{~cm}$ ). The nidus was not sampled endovascularly due to heightened procedural risk.

Following identification of the biopsy site, super selective microcatheterization of the desired arterial segment was performed under fluoroscopic guidance. Microcatheter position was confirmed with a focused angiogram. A platinum detachable coil was matched to the size of the parent vessel and deployed through the microcatheter contacting the vessel lumen only at the desired biopsy site. After 1 minute of luminal contact, the coil was resheathed and removed from the telescoping catheters which protected it from contacting other vessel segments. The removed coil was then cut and placed into a $3 \mathrm{~mL}$ LoBind Eppendorf microcentrifuge tube containing chilled cell dissociation buffer. New coils were selected, and coil embolization was carried out per routine practice to reduce blood flow to the AVM for open neurosurgical resection. Procedural steps of the endovascular biopsy may be viewed in Video 1.

\section{Computational Fluid Dynamic Modeling}

A Computational Fluid Dynamic (CFD) approach was used to compute the velocity field through the vasculature of interest and through the cardiac cycle. CFD simulations require accurate specification of boundary conditions, namely the luminal surface geometry and the inlet flow waveform through the cardiac cycle. The luminal contours were extracted with a level-set representation of the complex surface from the three-dimensional digital subtraction angiography (DSA) images acquired on either an Artis Q or Artis Zee biplane angiographic system (Siemens Healthineers AG, Forchheim, Germany). Images were transferred to a clinical post-processing workstation and reconstructed on a $512 \times 512$ matrix with a smooth HU kernel, resulting in 
approx. $0.8 \mathrm{~mm}$ actual resolution. The resulting reconstructed 3D-DSA volumes were exported and loaded onto a research workstation with the available CFD modeling research prototype (Siemens Healthineers AG, Forcheim, Germany). Prior to processing the site of endoluminal biopsy has been marked in the 3D volume in order to perform CFD analysis on the same vessel segment close to the nidus. ${ }^{10}$

The prototype CFD solver (Siemens Healthineers AG, Forchheim, Germany) uses a GPU-optimized Lattice-Boltzmann Method with a Multi-Relaxation Time approximation. ${ }^{11,12}$ In this approach, the incompressible Navier-Stokes equations are solved based on the Finite Volume Method and the pulsatile velocity field $(3 \mathrm{D}+\mathrm{t}$, i.e. in space and through the cardiac cycle) is computed on a Cartesian grid at $0.2 \mathrm{~mm}$ isotropic spatial resolution. This allows the determination of secondary hemodynamic descriptors such as the wall shear stress (WSS). The velocity waveform from Boegel et al. was used in this work and is adapted from the inflow velocity profile presented by Karmonik et al. which is based on 2D phase-contrast MRI measurements. ${ }^{13,14}$ In order to ensure convergence, flow computations were performed over two heartbeats with a time step of $5 \times 10^{-4} \mathrm{~s}$, and results were output every $0.05 \mathrm{~s}$. At all outflow boundaries the pressure was fixed to $0 \mathrm{~Pa}$. The blood density and viscosity were assumed to be $0.001 \mathrm{~g} / \mathrm{mm}^{\wedge} 3$ and $0.004 \mathrm{~Pa} \cdot \mathrm{s}$, respectively.

\section{Open neurosurgical sampling}

Patients were observed overnight in the intensive care unit and were taken to the neurosurgical operative suite the following day. All patients were placed under general anesthesia. A craniotomy was tailored to each patient with neuronavigational software in standard fashion and microsurgical resection was performed with aide of a Zeiss OPMI Pentero 
800 operative microscope (Carl Zeiss AG, Oberkochen, Germany). Direct microscopic visualization, including intraoperative indocyanine green angiography, ${ }^{15}$ and confirmation with neuronavigational software (BrainLab, Munich, Germany) identified feeding arteries, the nidus and draining veins of the AVM. To maintain 3D orientation, aneurysm clips and AVM micro clips (Aesculap, Inc., Center Valley, PA) of different sizes were placed under direct visualization onto feeding arteries of interest, including the endovascular biopsy site, and to mark the AVM nidus. Following AVM removal, the AVM was micro-dissected into the arterial segment from the biopsy site and nidus, and tissue samples from each site were placed in pre-oxygenated chilled Dulbecco's Modified Eagle Medium (DMEM, Thermo Fisher Scientific) and transported to the laboratory for subsequent dissociation.

\section{Cell preparation \& fluorescence-activated cell sorting}

For cell samples from endovascular biopsy, samples were vortexed for 30 seconds in cell dissociation buffer to liberate cells and the cut wire or coil was discarded. Cells were subsequently spun down and erythrocytes lysed with incubation in ACK lysing buffer (Thermo Fisher Scientific). Cells were washed with phosphate buffered saline (PBS). All tissues from open surgical resection were micro-dissected with a \#15 scalpel into roughly 1 x $1 \mathrm{~mm}$ cubes, and subsequently dissociated with $0.2 \%$ collagenase type 2 (Worthington Biochemical corporation, Lakewood, NJ). Erythrocytes were lysed with incubation in ACK lysing buffer, and cell suspension was filtered through a sterile $40 \mu \mathrm{m}$ filter to remove debris and washed with PBS.

Cell suspensions were resuspended in fluorescent activated cell sorting (FACS) buffer and stained with a modified protocol as we described ${ }^{8,9,16}$. Cells were stained with Alexa Fluor 
647-conjugated monoclonal anti-human CD31 antibody (BD Biosciences, San Jose, CA) and 4',6-diamidino-2-phenylindole (DAPI, Thermo Fisher Scientific). Cells were subsequently fluorescently sorted on a BD FACSAria II Flow Cytometer (BD Biosciences). Non-viable cells were excluded on basis of DAPI positivity, and endothelial enrichment was performed through positive selection of CD31-cells. Viable cells enriched for endothelium were therefore considered to be CD31-positive, DAPI-negative cells.

\section{Library preparation \& bulk RNA sequencing}

Library preparation and sequencing of samples were performed blinded to pathology. cDNA sequencing libraries were created directly with application of the SEQuoia complete RNA library prep kit (Biorad Laboratories, Inc, Hercules, CA) to whole cells as described by the manufacturer. Ribosomal RNA (rRNA) was depleted with proprietary technology post-library generation (Biorad Laboratories, Inc). All of the final libraries underwent quality control analysis with the Agilent D1000 ScreenTape System (Agilent Technologies, Santa Clara, CA) and were sequenced on an Illumina HiSeq 4000, single-end 50 base pair reads, multiplexed at 6 samples per lane.

\section{Quantitative polymerase chain reaction}

To confirm RNAseq results, quantitative polymerase chain reaction (qPCR) was performed on generated cDNA. A subset of five differentially expressed genes identified with highest score determined by $-\log _{10} \mathrm{p}$-value $*$ abs $(\beta)$ and a bioinformatic panel sensitive to activation of mitogen activated protein kinase (MAPK) signaling and predictive to response of MAPK/extracellular signal-regulated kinase (MEK) inhibitors were used for this analysis. ${ }^{17}$ 
Primers were designed using Fluidigm D3 assay design software (https://d3.fluidigm.com)

(Fluidigm Corporation, South San Francisco, CA), and assay IDs are available in eTable 1 in the Data Supplement. qPCR was performed using SBYR green (Roche Applied Science, Penzberg, Germany) on a LightCycler 480 II (Roche Applied Science). Relative gene expression was calculated using the delta-delta $\mathrm{C}_{\mathrm{T}}$ method using $\beta$-actin $(A C T B)$ as a housekeeping gene. Data is presented as mean \pm standard deviation for 4 biological replicates.

\section{Single Cell RNA Sequencing}

To confirm endothelial enrichment via FACS, we sorted individual CD31-positive, DAPI-negative cells acquired via endoluminal biopsy and prepared single cell RNA sequencing (scRNAseq) libraries using the Smart-seq2 protocol on 96-sorted cells as previously described. ${ }^{18}$ Final libraries underwent quality control analysis with the Agilent D1000 ScreenTape System (Agilent Technologies) and were sequenced on an Illumina HiSeq 4000 with paired-end 100 base pair reads.

\section{Data Analyses}

For sequencing data, sequencing quality was confirmed with FastQC and adaptors trimmed with Cutadapt. Reads were pseudoaligned to ENSEMBL GRCh38 transcriptome using Kallisto with 100 bootstrap samples. ${ }^{19}$ For scRNAseq data, gene count matrices were generated and analyzed with Seurat. ${ }^{20}$ Detected genes were defined as those with a transcript per million (TPM >1). Gene-level differential gene expression analysis was performed with Sleuth, and $\beta$ serves as an estimate of the fold-change as previously reported ${ }^{21}$. Genes with a false discovery rate (FDR) adjusted q-value $<0.05$ were considered to be differentially expressed. Gene 
ontology analyses were performed on differentially expressed genes with an FDR adjusted qvalue $<0.1$ using EnrichR. ${ }^{22}$ For in vivo flow modeling, Pearson correlation coefficients to deduce relationship of time-averaged wall shear stress with gene expression (expressed as transcripts per million) for each transcript across individuals. Genes with a correlation coefficient $\geq 0.6$ or $\leq-0.6$ were defined to be correlated genes. All data analyses and visualizations were performed with $\mathrm{R}$ version 3.6.3.

\section{Data Availability}

Anonymized data not published within this article will be made available by request from any qualified investigator.

\section{Results}

Endoluminal biopsy detects pathogenic and targetable molecular changes in brain arteriovenous malformations

Endoluminal biopsy was performed in all patients without complication (Figure 1A).

Patient demographic information is summarized Table 1. On average, 269.0 \pm 79.9 CD31postive, DAPI-negative viable cells were recovered per site (control: $309.2 \pm 86.6$ cells; AVM: $228.8 \pm 133.4$ cells) (Table 1). Using the FACS gating parameters utilized, single cell RNA sequencing confirmed enrichment of endothelial cells which comprise $71.9 \%$ of cells sampled based on canonical marker expression (eFigure 1A). RNAseq profiling of endoluminal biopsied cells identified 106 differentially expressed genes (DEGs) (FDR <0.05) (Figure 1B, eTable 2 in the Data Supplement). Pathway analysis showed DEGs were enriched for brain AVM pathogenic pathways - such as mitogen activated protein kinase (MAPK) signaling, ${ }^{5,6}$ angiogenesis (Rho- 
mediated cell motility, serum response factor activation, thrombin signaling), ${ }^{23,24}$ and inflammation (antigen processing) (Figure 1C). ${ }^{25}$ Ontological molecular function analysis confirmed enrichment of serine/threonine and protein kinase activity and binding to the small GTPases Ras and Rab (Figure 1C).

To confirm these findings, we next performed qRT-PCR analysis to verify DEGs and MAPK activation. We confirmed the differential expression of 5 genes (NBPF19, IFITM1, FBLX18, TNFRSR10C, and MARCHF8) among the 10 most differentially expressed genes (Figure 2A). We investigated MAPK activation with a gene panel independently shown to also predict response to MAPK/extracellular signal-regulated kinase (MEK) inhibitors in human cancer. ${ }^{17}$ This analysis demonstrated statistically significant upregulation of 7 out of 10 genes supporting MAPK activation (Figure 2B). Thus, endoluminal biopsy recovers primary cells that reflect molecular events in the malformed vasculature, enables direct molecular profiling and identifies potential therapeutic targets without the need for surgery.

Endoluminal biopsy closely approximates gene expression profiling from surgically excised arteriovenous malformations

We next sought to compare gene expression profiles obtained from endoluminally biopsied cells with surgically acquired tissues from the same patients - an important technical validation benchmark. We optimized a microsurgical technique to preserve $3 \mathrm{D}$ orientation of the bAVM and ensure sampling from an arterial segment contiguous with the biopsy site (Figure 3A). RNAseq analysis demonstrates that endoluminal biopsy detects the vast majority (83.3\%) of the genes identified in surgical tissue (Figure 3B). Gene expression profiles from endoluminal and surgically acquired cells correlated well genome-wide (Pearson $r=0.77$, Figure 3C), 
supporting good concordance between techniques. A strong correlation was also observed in RNAseq gene expression between feeding arteries and the nidus in surgically acquired bAVMs (Pearson $r=0.95$, Figure 3D). This supports that endoluminal sampling from more accessible sites, such as juxtanidal feeding arteries, is representative of molecular profiles in the AVM nidus without added procedural risk.

Incorporation of computational fluid dynamic modeling allows endoluminal biopsy to assay gene expression changes with alterations in cerebral blood flow

Hemodynamic alterations from cerebral blood flow (CBF) - such as wall shear stress are associated with vascular wall remodeling and risk of brain hemorrhage or onset of neurologic symptoms in bAVMs. ${ }^{26-28}$ To investigate how CBF alters the transcriptome, CFD modeling from cerebral angiograms was used to compute time averaged wall shear stress (TAWSS) at the site and time of endoluminal biopsy from cerebral angiograms (Figure 4A, Video 2). Across individuals, good correlation between TAWSS and RNAseq gene expression was seen in 11.0\% of transcripts (Figure 4B). We identified pathways positively or negatively correlated with TAWSS. Interestingly, this analysis showed enrichment of inflammatory and structural pathways that were negatively correlated with TAWSS (FDR <0.05) (Figure 4C). Ontological molecular function analyses showed enrichment in binding to RNA and protein kinases as well as to cytoskeletal elements - such as actin and cadherin - consistent with modulatory changes (Figure $4 C)$.

Embolization is an endovascular procedure in which an occlusive material - such as a platinum coil or liquid adhesive - reduces blood flow and wall shear stress to the bAVM. ${ }^{29}$ To confirm our predictions in living patients, we next evaluated the influence of flow-reductive 
embolization on gene expression in vivo. RNAseq profiling of endoluminal biopsies of the same bAVM arterial segment pre- and post-embolization confirmed differential expression of a minority of transcripts (7.3\%). Consistent with our correlative analyses, genes negatively correlated with shear stress, e.g., expression predicted to increase with lower shear stress, were upregulated in response to flow reduction (Figure 4D). In contrast, genes which correlated positively with shear stress were downregulated in response to reduced flow (Figure 4D). With integration of CFD modeling with gene expression profiling, endoluminal biopsy may facilitate future investigations to understand how alterations in CBF may influence cerebrovascular molecular changes in living patients.

\section{Discussion}

Here, we report the first in-human use of endoluminal biopsy as a means to molecularly profile bAVMs in living patients. We demonstrate that gene expression profiles detect pathogenic and potentially targetable molecular changes in bAVMs without need for invasive surgery. Importantly, there is good concordance in gene expression profiling obtained through endovascular biopsy when compared to surgically excised tissues - the gold standard. Our results further support involvement of aberrancies in Ras-MAPK signaling in adult bAVMs as previously reported. ${ }^{5,6}$ Targeted pathway inhibition - such as MEK inhibitors - reverse pathologic bAVM endothelial changes in vitro and in vivo and are being trialed in extracranial vascular malformations. ${ }^{6,7}$ Our results demonstrate that we are able to detect gene expression alterations which predict response to MEK inhibition in human cancers. ${ }^{17}$ Whether this predicts response of human bAVMs to MEK inhibition remains to be determined and is worthy of future investigation. 
Incorporation of CFD modeling showed an association of shear stress with gene expression changes and was confirmed by comparing pre- and post-embolization gene expression - an intervention modulating shear stress - in human patients in vivo. These analyses support prior in vitro works supporting a modulatory role of shear stress on endothelial inflammatory or cytoskeletal changes. ${ }^{29-31}$ Others have suggested physiologic shear stress is important for other structural elements, such as the blood-brain barrier. ${ }^{32,33}$ However, our observations were limited to focal segments of feeding arteries at the site of endoluminal biopsy complicating the interpretation and generalizability of these findings. The hemodynamic environment within bAVMs is immensely complex and presently poorly understood. ${ }^{34,35}$ Emerging technologies - such as 4D flow magnetic resonance imaging (MRI) or quantitative magnetic resonance angiography - are beginning to shed light on hemodynamic alterations within bAVMs. ${ }^{26,29,36}$ Endoluminal biopsies when performed in conjunction with CFD or quantitative MRI may serve as an experimental platform to begin to elucidate the interface between hemodynamic alterations and cellular-molecular changes within bAVMs.

The present study represents a first step in describing application of endoluminal biopsy to molecularly characterize bAVMs in living patients and is not without its limitations - most notably its small sample size and single center design. All findings should therefore be considered preliminary until further independent confirmation is performed at other centers to confirm generalizability. Our small sample size demonstrates initial feasibility but is underpowered to determine patient safety. In efforts to validate endoluminal biopsy comparisons were made with surgically sampled cells from the same patient. Despite minimizing the interval between endoluminal sampling and surgical resection $(<24$ hours), inflammatory and thrombotic pathways may have contributed to some of the observed differences between endoluminal and 
surgically sampled cells. Uncertainty surrounding safety with the endoluminal biopsy technique also prohibited collection from uninvolved cerebral arteries. Comparisons were performed between endothelium harvested from bAVM feeding arteries and patient-matched arteries outside the cerebrovasculature near the site of access, e.g., the iliac artery. Transcriptional variation between endothelium between different vascular beds has been reported, ${ }^{37,38}$ and may have contributed to observed differential gene expression. As safety profiles become better established, future endoluminal biopsy studies should be designed without embolization and perform comparisons with uninvolved cerebral vessels to confirm these findings.

A pivotal next step is performing larger, multicenter studies to characterize procedurerelated safety and complication profiles with endoluminal biopsy. A prevailing safety concern is that endoluminal biopsy would either promote thrombus formation or impair vascular structural integrity resulting in ischemic or hemorrhagic stroke, respectively. Risks for permanent neurologic complications with diagnostic cerebral angiography due to either ischemic or hemorrhagic stroke is $<1 \%$. ${ }^{39,40}$ Preclinical testing has shown that deployment of a detachable coil to acquire cells does not lead to angiographic or microscopic damage to the sampled artery. ${ }^{8}$ We have also adopted a tiered strategy to mitigate risks of ischemia: 1) systemic heparinization prior to biopsy; 2) avoidance of flow limitation with coil deployment; 3) limitation of endothelial contact time to 1-minute; and 4) live fluoroscopic monitoring for potential thrombus formation with either coil deployment or immediately following biopsy. With our growing institutional experience, we have yet to encounter a procedural complication associated with endoluminal biopsy applying these strategies. However, the additive risks to performing endoluminal biopsy with a diagnostic cerebral angiogram presently remain unquantified in human patients. 
Diagnostic molecular genomics continues to evolve in clinical laboratories, especially within neuro-oncology. Except for the PCR panel predictive of responses to MEK inhibition, ${ }^{17}$ the present manuscript employs RNAseq and computational tools which would be difficult to employ in larger clinical settings. Ongoing investigations have begun to identify molecular diversity in bAVMs and how it relates to divergent natural histories. ${ }^{5,6,41}$ As candidate gene lists become better defined, downstream analytics should employ more-targeted gene panels either predictive of therapeutic response or risk for rupture, and we are currently iterating to produce a more targeted test not reliant on genome-wide or exome-wide sequencing.

Clinical decisions to treat or monitor vascular malformations are currently informed with imperfect radiologic or clinical surrogates which may result in divergent natural histories. ${ }^{42}$ With further development of endoluminal biopsy, however, it may be possible to directly categorize or serially monitor vascular malformations or other vascular diseases molecularly - much like a tumor. Medical therapy has a favorable safety profile in randomized controlled trials, ${ }^{3}$ and endoluminal biopsy could theoretically help identify candidates for targeted precision based approaches that have shown promise in experimental models. ${ }^{6,7}$ Whether endoluminal biopsy will facilitate trials of precision medical therapies in human patients or better identify vascular malformations at greatest risk of bleeding requires further investigation.

Supplement --http://links.lww.com/WNL/B817

Video1 --http://links.lww.com/WNL/B818

Video2 --http://links.lww.com/WNL/B819 


\section{References}

1. Solomon RA, Connolly ES, Jr. Arteriovenous Malformations of the Brain. N Engl J Med 2017;376:1859-1866.

2. Lawton MT, Rutledge WC, Kim H, et al. Brain arteriovenous malformations. Nat Rev Dis Primers 2015;1:15008.

3. Mohr JP, Overbey JR, Hartmann A, et al. Medical management with interventional therapy versus medical management alone for unruptured brain arteriovenous malformations (ARUBA): final follow-up of a multicentre, non-blinded, randomised controlled trial. Lancet Neurol 2020;19:573-581.

4. Winkler EA, Lu AY, Raygor KP, et al. Defective vascular signaling \& prospective therapeutic targets in brain arteriovenous malformations. Neurochem Int 2019;126:126-138.

5. Hong T, Yan Y, Li J, et al. High prevalence of KRAS/BRAF somatic mutations in brain and spinal cord arteriovenous malformations. Brain 2019;142:23-34.

6. Nikolaev SI, Vetiska S, Bonilla X, et al. Somatic Activating KRAS Mutations in Arteriovenous Malformations of the Brain. N Engl J Med 2018;378:250-261.

7. Fish JE, Flores Suarez CP, Boudreau E, et al. Somatic Gain of KRAS Function in the Endothelium Is Sufficient to Cause Vascular Malformations That Require MEK but Not PI3K Signaling. Circ Res 2020;127:727-743.

8. Cooke DL, Bauer D, Sun Z, et al. Endovascular biopsy: Technical feasibility of novel endothelial cell harvesting devices assessed in a rabbit aneurysm model. Interv Neuroradiol $2015 ; 21: 120-128$. 
9. Cooke DL, McCoy DB, Halbach VV, et al. Endovascular Biopsy: In Vivo Cerebral Aneurysm Endothelial Cell Sampling and Gene Expression Analysis. Transl Stroke Res 2018;9:20-33.

10. Suzuki T, Ioan Nita C, Rapaka S, et al. Verification of a research prototype for hemodynamic analysis of cerebral aneurysms. Annu Int Conf IEEE Eng Med Biol Soc 2016;2016:2921-2924.

11. D'Humieres D, Ginzburg I, Krafczyk M, Lallemand P, Luo LS. Multiple-relaxation-time lattice Boltzmann models in three dimensions. Philos Trans A Math Phys Eng Sci 2002;360:437451.

12. Nita CI, Suzuki T, Itu LM, et al. An Automated Workflow for Hemodynamic Computations in Cerebral Aneurysms. Comput Math Methods Med 2020;2020:5954617.

13. Boegel M, Gehrisch S, Redel T, et al. Patient-individualized boundary conditions for CFD simulations using time-resolved 3D angiography. Int J Comput Assist Radiol Surg 2016;11:1061-1069.

14. Karmonik C, Klucznik R, Benndorf G. Blood flow in cerebral aneurysms: comparison of phase contrast magnetic resonance and computational fluid dynamics--preliminary experience. Rofo 2008;180:209-215.

15. Foster $\mathrm{CH}$, Morone PJ, Tomlinson SB, Cohen-Gadol AA. Application of Indocyanine Green During Arteriovenous Malformation Surgery: Evidence, Techniques, and Practical Pearls. Front Surg 2019;6:70.

16. Sun Z, Lawson DA, Sinclair E, et al. Endovascular biopsy: Strategy for analyzing gene expression profiles of individual endothelial cells obtained from human vessels(). Biotechnol Rep (Amst) 2015;7:157-165. 
17. Wagle MC, Kirouac D, Klijn C, et al. A transcriptional MAPK Pathway Activity Score (MPAS) is a clinically relevant biomarker in multiple cancer types. NPJ Precis Oncol 2018;2:7. 18. Picelli S, Faridani OR, Bjorklund AK, Winberg G, Sagasser S, Sandberg R. Full-length RNA-seq from single cells using Smart-seq2. Nat Protoc 2014;9:171-181.

19. Bray NL, Pimentel H, Melsted P, Pachter L. Near-optimal probabilistic RNA-seq quantification. Nat Biotechnol 2016;34:525-527.

20. Stuart T, Butler A, Hoffman P, et al. Comprehensive Integration of Single-Cell Data. Cell 2019;177:1888-1902 e1821.

21. Pimentel H, Bray NL, Puente S, Melsted P, Pachter L. Differential analysis of RNA-seq incorporating quantification uncertainty. Nat Methods 2017;14:687-690.

22. Kuleshov MV, Jones MR, Rouillard AD, et al. Enrichr: a comprehensive gene set enrichment analysis web server 2016 update. Nucleic Acids Res 2016;44:W90-97.

23. Franco CA, Blanc J, Parlakian A, et al. SRF selectively controls tip cell invasive behavior in angiogenesis. Development 2013;140:2321-2333.

24. van Nieuw Amerongen GP, Koolwijk P, Versteilen A, van Hinsbergh VW. Involvement of RhoA/Rho kinase signaling in VEGF-induced endothelial cell migration and angiogenesis in vitro. Arterioscler Thromb Vasc Biol 2003;23:211-217.

25. Hauer AJ, Kleinloog R, Giuliani F, et al. RNA-Sequencing Highlights Inflammation and Impaired Integrity of the Vascular Wall in Brain Arteriovenous Malformations. Stroke 2020;51:268-274.

26. Chang $\mathrm{W}$, Loecher MW, Wu Y, et al. Hemodynamic changes in patients with arteriovenous malformations assessed using high-resolution 3D radial phase-contrast MR angiography. AJNR Am J Neuroradiol 2012;33:1565-1572. 
27. Chen X, Cooke DL, Saloner D, et al. Higher Flow Is Present in Unruptured Arteriovenous Malformations With Silent Intralesional Microhemorrhages. Stroke 2017;48:2881-2884.

28. Winkler EA, Birk H, Burkhardt JK, et al. Reductions in brain pericytes are associated with arteriovenous malformation vascular instability. J Neurosurg 2018;129:1464-1474.

29. Alaraj A, Shakur SF, Amin-Hanjani S, et al. Changes in wall shear stress of cerebral arteriovenous malformation feeder arteries after embolization and surgery. Stroke 2015;46:12161220.

30. DeStefano JG, Xu ZS, Williams AJ, Yimam N, Searson PC. Effect of shear stress on iPSC-derived human brain microvascular endothelial cells (dhBMECs). Fluids Barriers CNS 2017;14:20.

31. Inglebert M, Locatelli $\mathrm{L}$, Tsvirkun $\mathrm{D}$, et al. The effect of shear stress reduction on endothelial cells: A microfluidic study of the actin cytoskeleton. Biomicrofluidics 2020;14:024115.

32. Garcia-Polite F, Martorell J, Del Rey-Puech P, et al. Pulsatility and high shear stress deteriorate barrier phenotype in brain microvascular endothelium. J Cereb Blood Flow Metab 2017;37:2614-2625.

33. Vatine GD, Barrile R, Workman MJ, et al. Human iPSC-Derived Blood-Brain Barrier Chips Enable Disease Modeling and Personalized Medicine Applications. Cell Stem Cell 2019;24:995-1005 e1006.

34. Chang W, Huang M, Chien A. Emerging techniques for evaluation of the hemodynamics of intracranial vascular pathology. Neuroradiol J 2015;28:19-27. 
35. Jain MS, Do HM, Massoud TF. Computational Network Modeling of Intranidal Hemodynamic Compartmentalization in a Theoretical Three-Dimensional Brain Arteriovenous Malformation. Front Physiol 2019;10:1250.

36. Markl M, Wu C, Hurley MC, et al. Cerebral arteriovenous malformation: complex 3D hemodynamics and 3D blood flow alterations during staged embolization. J Magn Reson Imaging 2013;38:946-950.

37. Jambusaria A, Hong Z, Zhang L, et al. Endothelial heterogeneity across distinct vascular beds during homeostasis and inflammation. Elife 2020;9.

38. Kalucka J, de Rooij L, Goveia J, et al. Single-Cell Transcriptome Atlas of Murine Endothelial Cells. Cell 2020;180:764-779 e720.

39. Dawkins AA, Evans AL, Wattam J, et al. Complications of cerebral angiography: a prospective analysis of 2,924 consecutive procedures. Neuroradiology 2007;49:753-759. 40. Willinsky RA, Taylor SM, TerBrugge K, Farb RI, Tomlinson G, Montanera W. Neurologic complications of cerebral angiography: prospective analysis of 2,899 procedures and review of the literature. Radiology 2003;227:522-528.

41. Li H, Nam Y, Huo R, et al. De Novo Germline and Somatic Variants Convergently Promote Endothelial-to-Mesenchymal Transition in Simplex Brain Arteriovenous Malformation. Circ Res 2021.

42. Stapf C, Mast H, Sciacca RR, et al. Predictors of hemorrhage in patients with untreated brain arteriovenous malformation. Neurology 2006;66:1350-1355. 


\section{Figure legends}

\section{Figure 1 - Endoluminal biopsy detects pathologic gene expression changes in brain}

arteriovenous malformations. (A) Endoluminal biopsy is achieved through image-guided placement of a cerebral angiography microcatheter into the lumen of an arteriovenous malformation (AVM). A platinum coil is then deployed contacting and removing the cellular lining of the artery. The coil is removed, and subsequent fluorescent-activated cell sorting enriches for endothelium. Figure courtesy of Kenneth Xavier Probst. (B) Volcano plot of RNAseq gene expression from AVM and patient-matched uninvolved vessel. FDR, false discovery rate. Beta, effect size. (C) Bar graph showing ontological analysis of differentially expressed genes. Orange, signaling pathways. Blue, molecular function. 


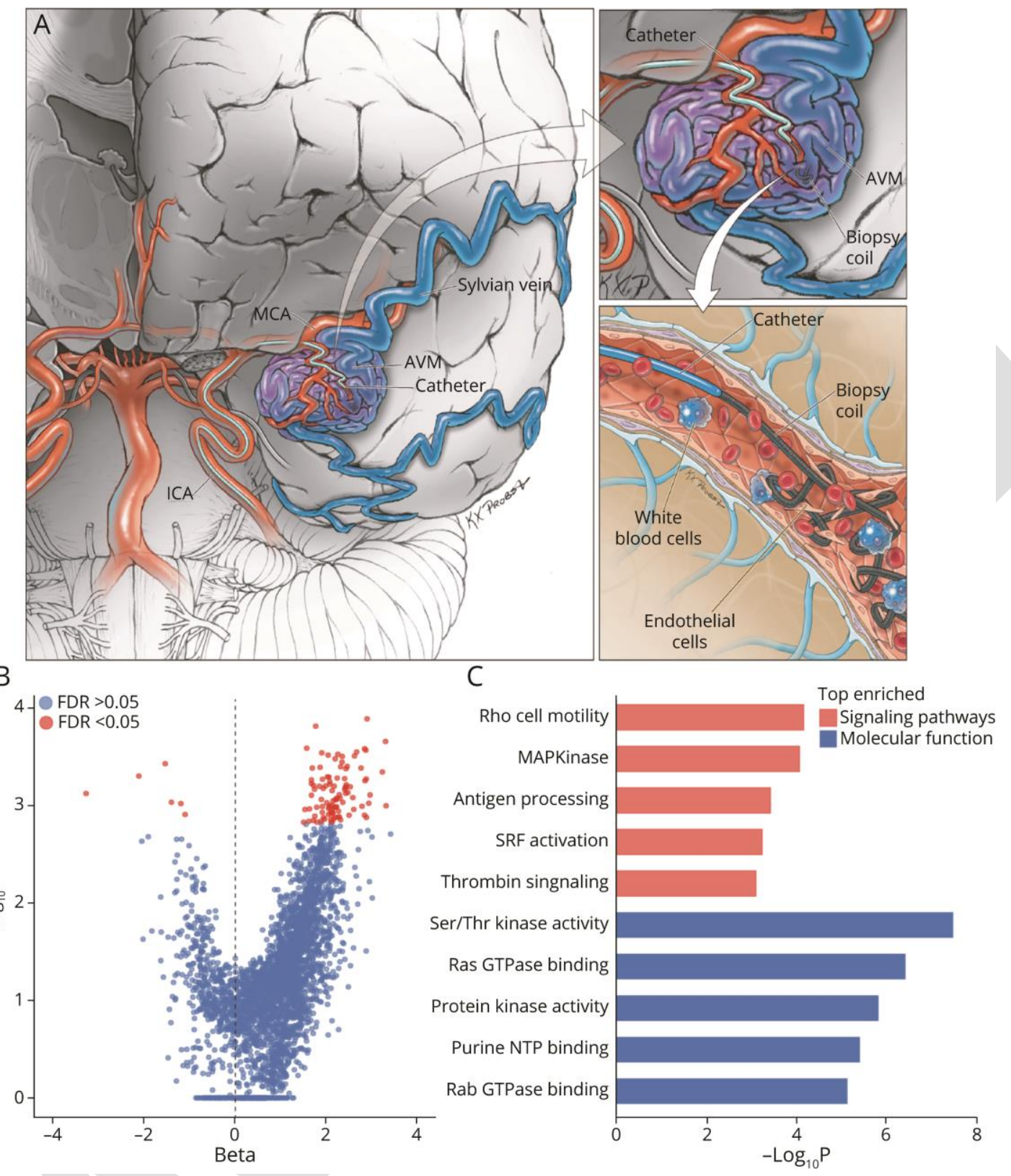

Copyright @ 2022 The Author(s). Published by Wolters Kluwer Health, Inc. on behalf of the American Academy of Neurology. 
Figure 2 - Validation of endoluminal biopsy-detected brain arteriovenous malformation gene expression changes. (A-B) Bar graph showing quantitative reverse transcription polymerase chain reaction expression values of differentially expressed genes (A) and gene panel for mitogen activated protein kinase (MAPK) activation $(\mathbf{B})$. Values expressed as $\log _{2}$ fold change $\left(\log _{2} \mathrm{FC}\right)$ in gene expression in arteriovenous malformation versus patient-matched controls. Orange, increased expression in arteriovenous malformation. Blue, decreased expression in arteriovenous malformation. $N D$, not detected. Data presented as mean \pm standard error of the mean.

A. Differential expressed genes
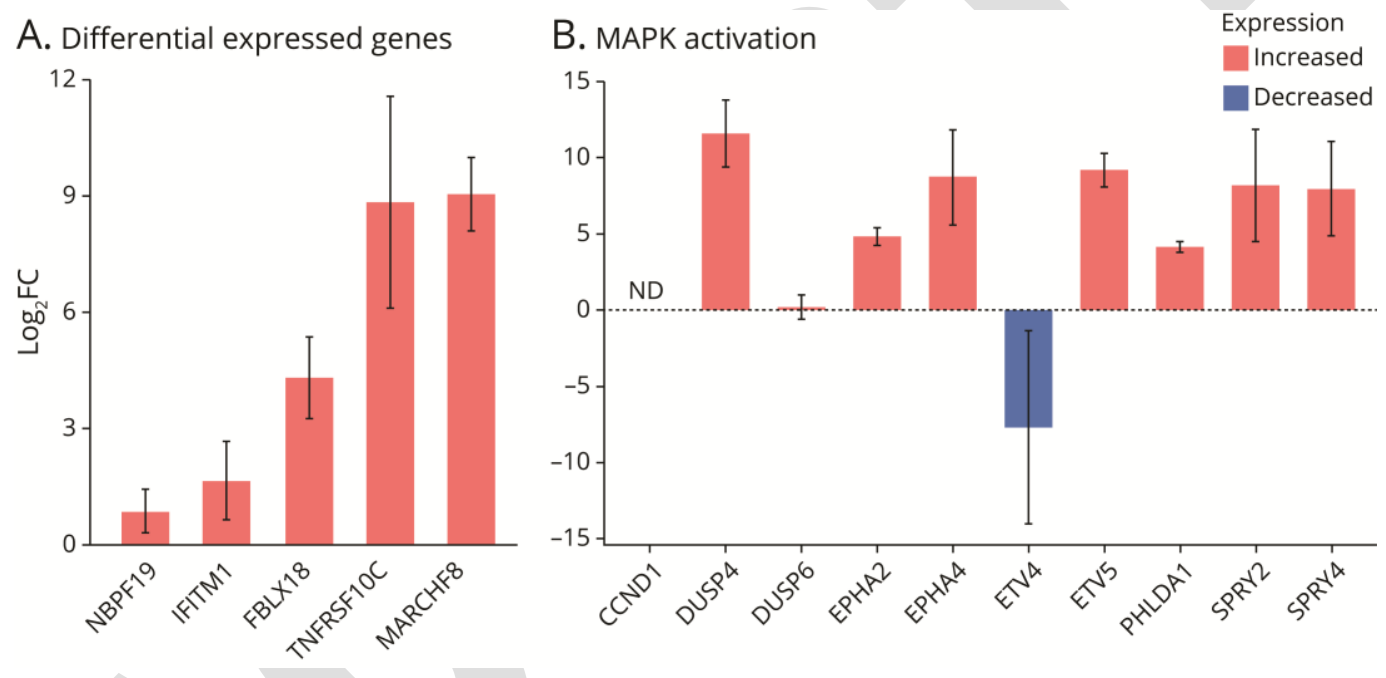
Figure 3 - Endoluminal biopsy gene expression profiles show good concordance with open surgical sampling. (A) Representative intraoperative microscopy showing microsurgical resection of a brain arteriovenous malformation (bAVM). Three-dimensional orientation is preserved with different sized aneurysm clips. *, draining vein. Arrow, feeding artery. (B) Venn diagram comparison of detected genes (transcript per million > 1) from endoluminal (orange) and open surgical sampling (blue). (C) Scatter plot showing correlation of gene expression from endovascular and surgical sampling from contiguous arteriovenous malformation arterial segment. TPM, transcripts per million; r, Pearson correlation coefficient. (D) Scatter plot showing correlation of gene expression of AVM feeding artery and nidus from surgically sampling. TPM, transcripts per million; r, Pearson correlation coefficient. 
A

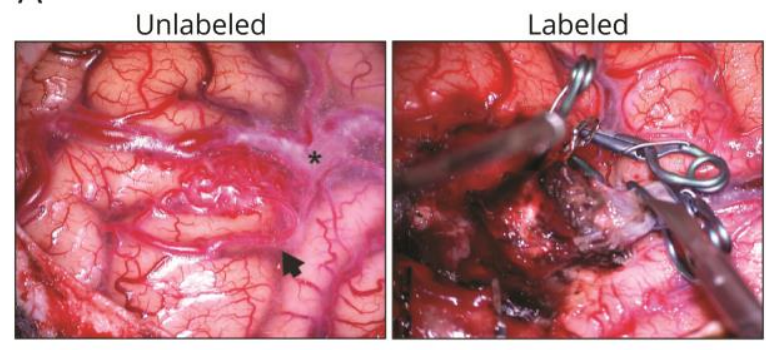

B. Sampling method Oopen surgery

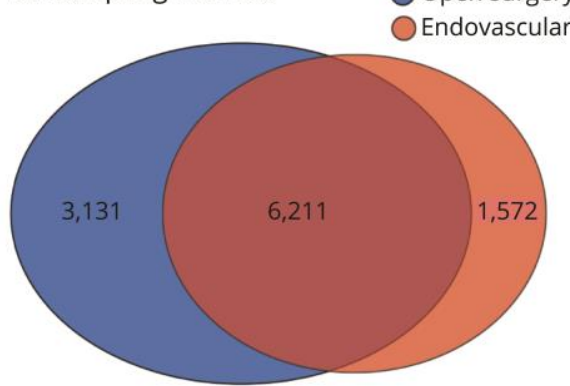

\section{Artery}

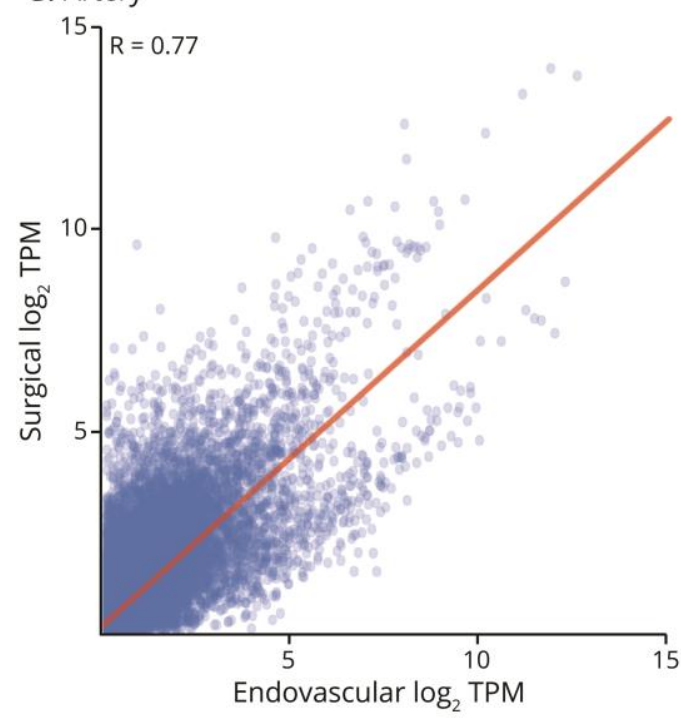

D. Surgical tissue

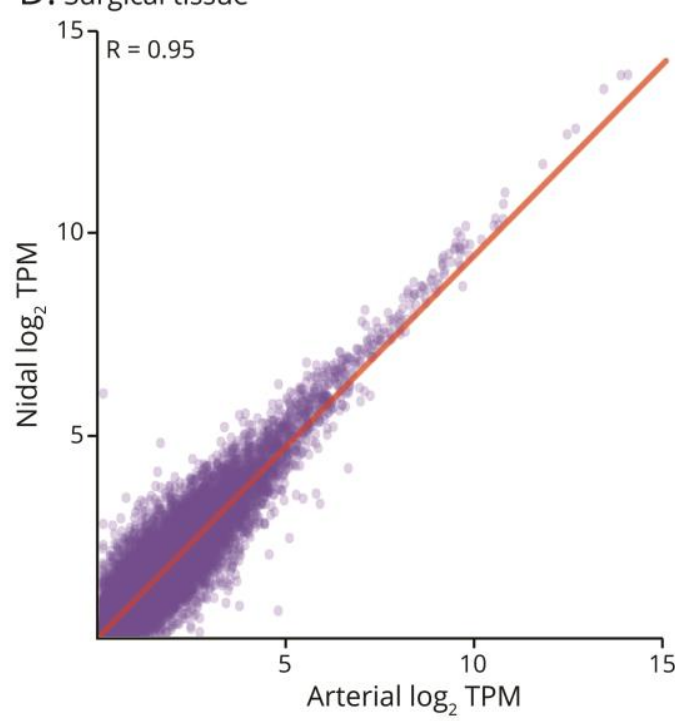


Figure 4 -Flow-related shear stress influences arteriovenous malformation gene expression.

A. Three-dimensional rotational angiography with computational fluid dynamic shear stress modeling of cerebral angiogram at site of biopsy in arteriovenous malformation (AVM) artery. Injection, vertebral artery. TAWSS, time averaged wall shear stress. Pa, pascal. B. Gene expression: Pie chart showing flow-correlated (orange) and non-correlated (blue) gene expression. C. Top enriched: Ontological analysis of signaling pathways (orange) and molecular function (blue) of flow-correlated genes. Left, negatively correlated. Right, positively correlated. Negatively correlated pathways are predicted to be activated with lower shear stress. Positively correlated pathways are predicted to be activated with higher shear stress. D. $\Delta$ Postembolization: Boxplot of gene expression changes $(\Delta)$ following flow-reductive embolization. Orange, genes negatively correlated with shear stress. Blue, genes positively correlated with shear stress. *, $\mathrm{p}<0.05$. 


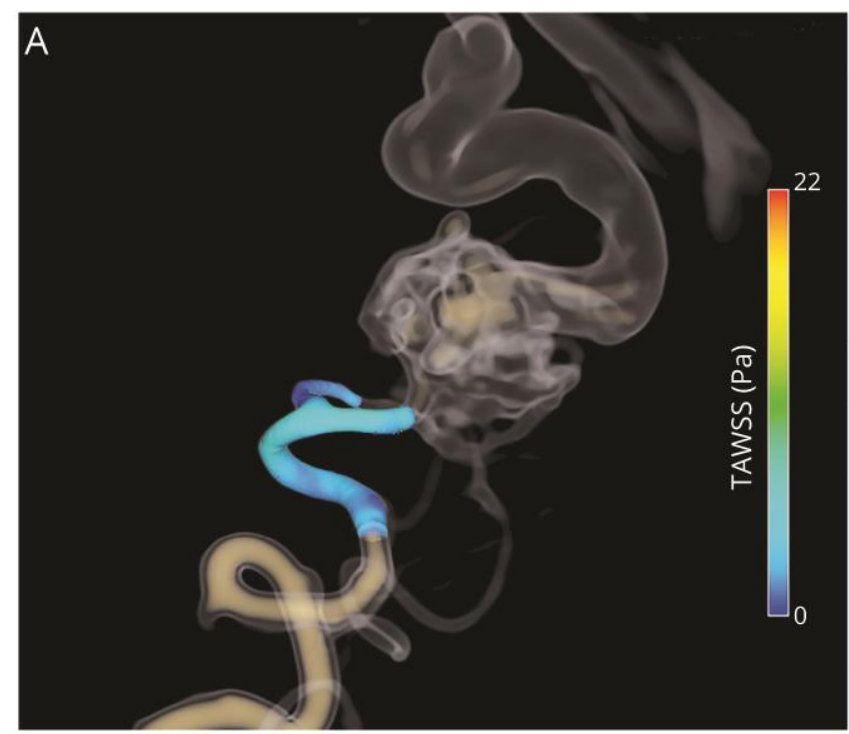

C. Top enriched

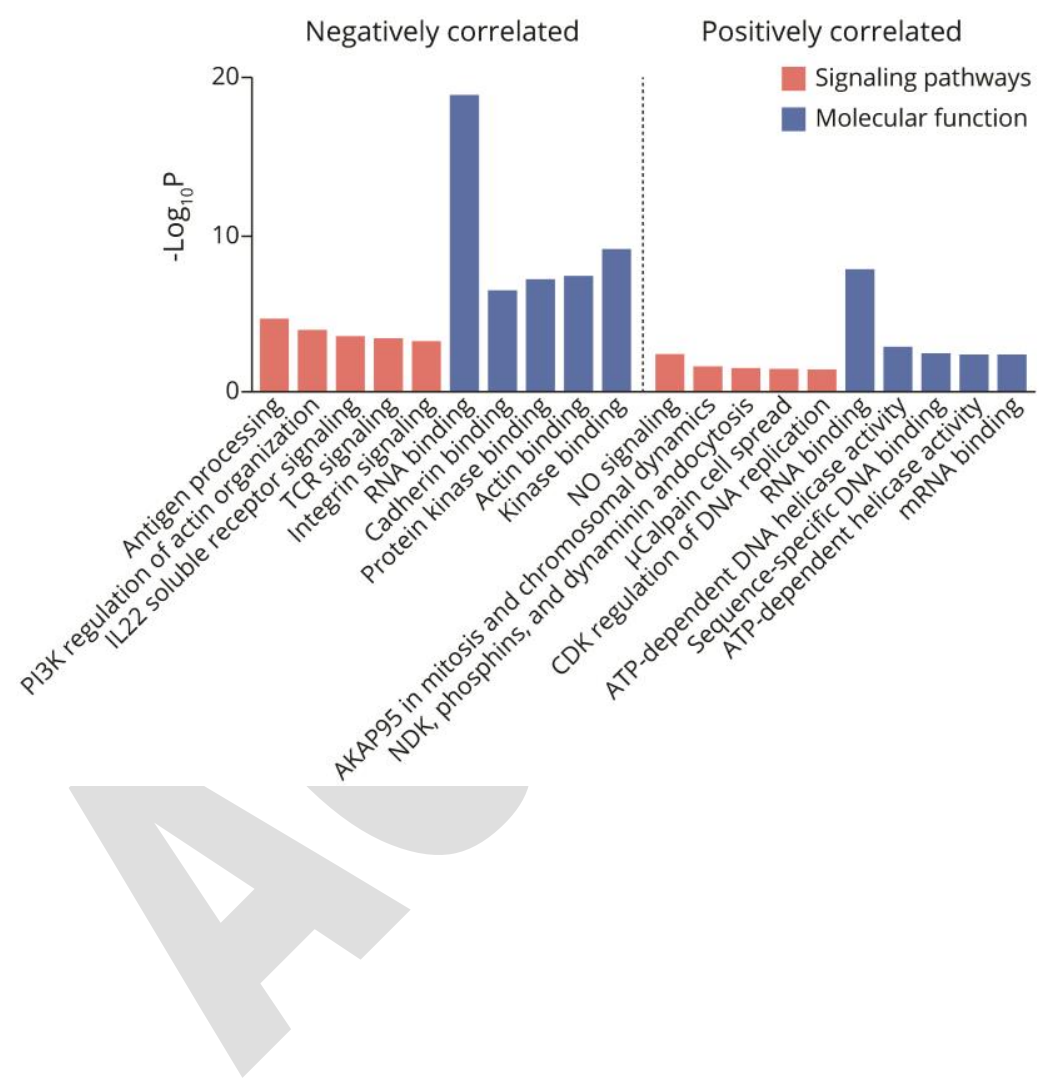

B. Gene expression

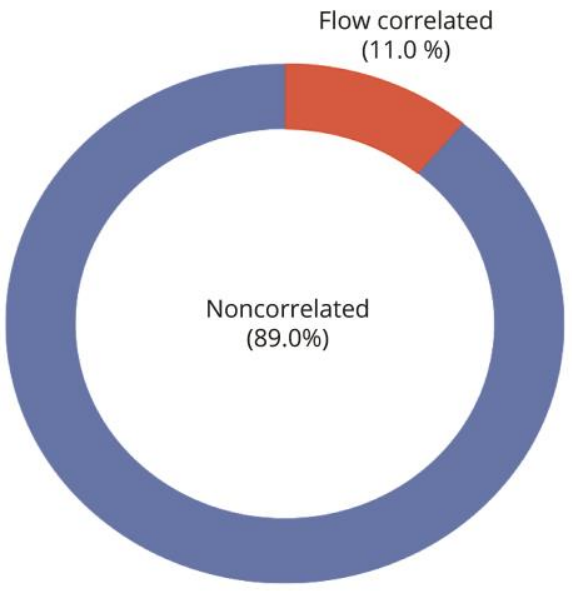

D. $\Delta$ Postembolization Flow correlation Negative

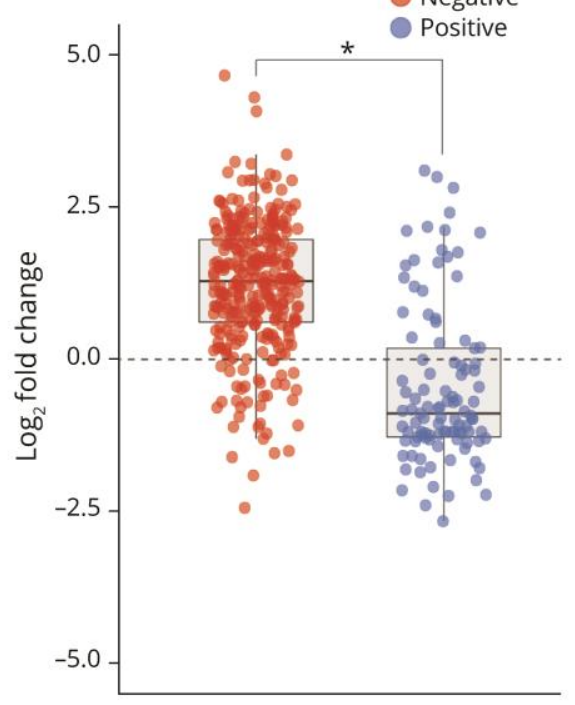


Table 1 - Patient demographics and cellular yield with endoluminal sampling of brain arteriovenous malformations.

\begin{tabular}{|c|c|c|c|c|c|c|c|}
\hline \multirow[t]{2}{*}{ Age } & \multirow[t]{2}{*}{ Gender } & \multirow[t]{2}{*}{ Location } & \multirow[t]{2}{*}{$\begin{array}{c}\text { SM } \\
\text { grade }\end{array}$} & \multicolumn{2}{|c|}{$\begin{array}{c}\text { Endoluminal cell yield } \\
\text { (\# cells) }\end{array}$} & \multicolumn{2}{|c|}{$\begin{array}{l}\text { Surgical cell yield } \\
\text { (\# cells) }\end{array}$} \\
\hline & & & & Iliac artery & AVM & AVM artery & AVM nidus \\
\hline 31 & $\mathrm{~F}$ & $\begin{array}{l}\text { Parieto- } \\
\text { occipital }\end{array}$ & 1 & 140 & 14 & 1052 & 9726 \\
\hline 49 & M & $\begin{array}{l}\text { Parieto- } \\
\text { occipital }\end{array}$ & 1 & 528 & 57 & 828 & 2782 \\
\hline 48 & M & Temporal & 4 & 206 & 244 & 157 & 282 \\
\hline 23 & $\mathrm{~F}$ & Occipital & 2 & 363 & 600 & $\mathrm{NC}$ & $\mathrm{NC}$ \\
\hline & & & Mean: & $\begin{array}{l}309.2 \pm \\
86.6\end{array}$ & $\begin{array}{c}228.8 \pm \\
133.4\end{array}$ & $\begin{array}{c}679.0 \pm \\
268.9\end{array}$ & $\begin{array}{c}4263.3 \pm \\
2825.1\end{array}$ \\
\hline
\end{tabular}

NC, not collected. 


\section{Supplementary Video Legends}

Video 1 - Endoluminal biopsy. Video depicting technique for endoluminal biopsy with digital subtraction angiography of brain arteriovenous malformation.

Video 2 - Vessel wall shear stress with cardiac cycle. Video showing computational fluid dynamic modeling of vessel wall shear stress over one cardiac cycle at site of endoluminal biopsy in feeding artery of brain arteriovenous malformation. 


\section{Neurology}

Endoluminal Biopsy for Molecular Profiling of Human Brain Vascular Malformations

Ethan Andrew Winkler, David Wu, Eugene Gil, et al.

Neurology published online February 10, 2022

DOI 10.1212/WNL.0000000000200109

This information is current as of February 10, 2022

Updated Information \&

Services

Subspecialty Collections

Errata

Permissions \& Licensing

Reprints including high resolution figures, can be found at: http://n.neurology.org/content/early/2022/02/10/WNL.0000000000200109.f ull

This article, along with others on similar topics, appears in the following collection(s):

Arteriovenous malformation

http://n.neurology.org/cgi/collection/arteriovenous_malformation

Gene expression studies

http://n.neurology.org/cgi/collection/gene_expression_studies

Intracerebral hemorrhage

http://n.neurology.org/cgi/collection/intracerebral_hemorrhage

Stroke in young adults

http://n.neurology.org/cgi/collection/stroke_in_young_adults

An erratum has been published regarding this article. Please see next page or:

/content/early/2023/01/30/WNL.0000000000201493.full.pdf

Information about reproducing this article in parts (figures,tables) or in its entirety can be found online at:

http://www.neurology.org/about/about_the_journal\#permissions

Information about ordering reprints can be found online:

http://n.neurology.org/subscribers/advertise

Neurology ${ }^{\circledR}$ is the official journal of the American Academy of Neurology. Published continuously since 1951, it is now a weekly with 48 issues per year. Copyright Copyright (C) 2022 The Author(s). Published by Wolters

Kluwer Health, Inc. on behalf of the American Academy of Neurology.. All rights reserved. Print ISSN:

0028-3878. Online ISSN: 1526-632X.

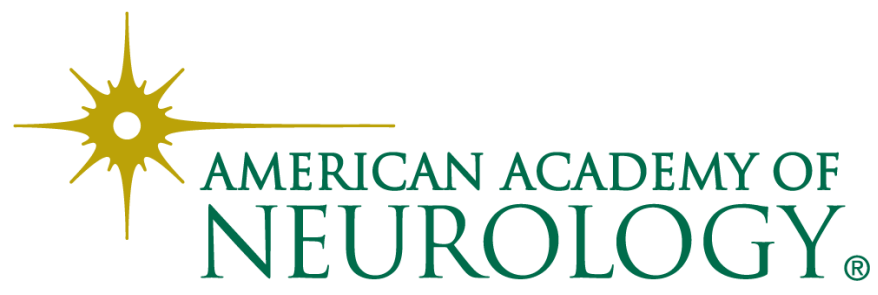




\section{Neurology Publish Ahead of Print \\ DOI: 10.1212/WNL.0000000000201493}

CORRECTION \& REPLACEMENT

Endoluminal Biopsy for Molecular Profiling of Human Brain Vascular Malformations In the Research Article "Endoluminal Biopsy for Molecular Profiling of Human Brain Vascular Malformations" by Winkler et al., ${ }^{1}$ the fifth label on the y axis of the graph shown in figure $1 \mathrm{C}$ should read "Thrombin signaling." The figure has been replaced by a corrected version. The original version with the change highlighted is available from a link in the corrected article. The editorial staff regrets the error.

\section{REFERENCE}

${ }^{1}$ Winkler E, Wu D, Gil E, et al. Endoluminal Biopsy for Molecular Profiling of Human Brain Vascular Malformations. Neurology. 2022;98:e1637-e1647.

Neurology ${ }^{\circledR}$ Published Ahead of Print articles have been peer reviewed and accepted for publication. This manuscript will be published in its final form after copyediting, page composition, and review of proofs. Errors that could affect the content may be corrected during these processes. 